Pacific Journal of Mathematics

THE STRUCTURE OF GALOIS CONNECTIONS 


\section{THE STRUCTURE OF GALOIS CONNECTIONS}

\section{Zahava ShMuely}

This paper deals with Galois connections between two partially ordered sets (posets) $A, B$. The first sections are devoted to the construction of all Galois connections between $A$ and $B$. The last sections deal with properties of $A \otimes B$, the set of mappings $T: A \rightarrow$ $B$ which "participate" in a Galois connection between $A$ and $B$, with the pointwise partial order.

Every Galois connection between two posets $A, B$ can be uniquely extended to a Galois connection between $\nu(A)$ and $\nu(B)$, the completions by cuts of $A, B$ resp., and $A \otimes B$ is characterized as a subset of $\nu(A) \otimes \nu(B)$. As an application we get: The completion by cuts of a residuated groupoid (semigroup) is a residuated groupoid (semigroup, resp.). The completion by cuts of a Brouwerian lattice is a Brouwerian lattice. The completion by cuts of a relation algebra is a relation algebra. When $A$ and $B$ are complete lattices, $A \otimes B$ is isomorphic to a certain set of semi-ideals of $A \times B$. This yields a procedure for constructing all Galois connections between any two posets. By dualization all sup-preserving and inf-preserving mappings are determined.

Bounded posets $A, B$ are embedded in $A \otimes B$ in a peculiar way. $A \otimes B$ is a completely distributive, complete (Boolean) lattice iff $A$ and $B$ are completely distributive, complete (Boolean, resp.) lattices. Formal properties of $\otimes$ as a binary operation on bounded posets are investigated. In particular $A \otimes 2^{B} \cong A^{B}$ when $A$ is a complete lattice, implying $A \otimes B^{C} \cong$ $A^{C} \otimes B \cong(A \otimes B)^{C}$ when $A, B$ are complete lattices and $C$ is a poset. In certain respects, the behavior of $A \otimes B$ as a product of $A$ and $B$ resembles that of the tensor product of linear spaces. ${ }^{1}$

1. In the following, $A, B, C$ denote partially ordered sets (posets). $A^{D}$ is the dual $A$. $A$ is bounded if it contains universal elements 0,1 with $0 \leq p$ $\leq 1$ for every $p \in A .2$ is the poset $\{0,1\}$ with $0<1$. A mapping $T: A \rightarrow B$ is isotone or order-preserving (antitone) whenever $p_{1} \leq p_{2}$ in $A$ implies $T\left(p_{1}\right)$ $\leq T\left(p_{2}\right)\left(T\left(p_{1}\right) \geq T\left(p_{2}\right)\right)$. Isomorphism here means order-isomorphism. $A$ is a complete lattice if every set $\left\{x_{\alpha}\right\} \subseteq A$ has a l.u.b., $\vee_{\alpha} x_{\alpha}$, and a g.l.b., $\wedge_{\alpha} x_{\alpha}$. A complete lattice is completely distributive whenever

$$
\wedge_{\alpha \in \Omega} \bigvee_{\beta \in B_{\alpha}} x_{\alpha \beta}=\bigvee_{\phi \in \Pi B_{\alpha}} \wedge_{\alpha \in \Omega} x_{\alpha \phi(\alpha)}
$$


holds for every set $\left\{x_{\alpha \beta}\right\} \subseteq A . X \subseteq A$ is a (dual) semi-ideal of $A$ if $(y \geq x)$ $y \leq x$ and $x \in X$ implies $y \in X$.

For $X \subseteq A$ let $X^{+}, X^{\wedge} \subseteq A$ be defined by $X^{+}=\{a \mid a \leq x$ for every $x \in$ $X\} ; X^{\wedge}=\{a \mid a \geq x$ for every $x \in X\}$. Adjoining 0 to $A$ if necessary put ([1] p. 126): $\nu(A)=\left\{X^{\wedge+} \mid X \subseteq A\right\} . \nu(A)$, partially ordered by inclusion is the completion by cuts of $A . a \rightarrow\{a\}^{\wedge+}$ embeds $A$ in the complete lattice $\nu(A)$.

$A \times B$ is the Cartesian product of $A$ and $B$, partially ordered by $\left(a_{1}, b_{1}\right)$ $\leq\left(a_{2}, b_{2}\right)$ if $a_{1} \leq a_{2}, b_{1} \leq b_{2} . A^{B}$ is the set of all isotone mappings $T: B \rightarrow A$ partly ordered by the pointwise partial order, i.e., $T_{1} \leq T_{2} \in A^{B}$ if $T_{1}(q) \leq$ $T_{2}(q)$ for each $q \in B$.

A pair $\langle T, G\rangle$ of mappings $T: A \rightarrow B, G: B \rightarrow A$ is a Galois connection between $A$ and $B$ [10] if: (i) $T, G$ are antitone; (ii) for each $p \in A, G T(p) \geq$ $p$, for each $q \in B, T G(q) \geq q$. Galois connections are treated in [1], [4], [10], [12] and used in many disciplines of mathematics (see for example [14], [16]). $A \otimes B$ denotes the set of mappings $T: A \rightarrow B$ for which a (necessarily unique) mapping denoted by $T^{*}: B \rightarrow A$ exists, such that $\left\langle T, T^{*}\right\rangle$ is a Galois connection between $A$ and $B . A \otimes B$ is given the pointwise partial order. If $A, B$ are complete lattices then ([12]):

$$
T \in A \otimes B \text { iff: (i) } T(0)=1 \text {; (ii) } T\left(\vee_{\alpha} a_{\alpha}\right)=\wedge_{\alpha} T\left(a_{\alpha}\right)
$$

for every set $\left\{a_{\alpha}\right\} \subseteq A$,

i.e., $T$ is a complete join-morphism on $A$ into $B^{D}$. Galois connections are also studied in a dualized form. The mapping $T: A \rightarrow B$ is residuated (residual) if $T \in A \otimes B^{D}\left(T \in A^{D} \otimes B\right)$. The semigroup of residuated mappings, where multiplication is function composition, is studied and used by many authors (see [2], [3], [5], [6], [8], [9]). By (1), residuated (residual) mappings on complete lattices are exactly the sup-(inf-) preserving mappings.

A procedure for constructing all Galois connections between completely distributive, complete Boolean lattices $A=2^{M}, B=2^{N}$ was given by Birkhoff ([1] p. 122). Raney [13], presented a procedure for constructing Galois connections between complete lattices. By his procedure all Galois connections between completely distributive, complete lattices, can be constructed.

2. In this section we prove:

THEOREM 1.2. Every Galois connection $\left\langle T, T^{*}\right\rangle$ between two posets $A$ 
and $B$ can be uniquely extended to a Galois connection $\left\langle\tilde{T}, \tilde{T}^{*}\right\rangle$ between $\nu(A)$ and $\nu(B)$, the completions by cuts of $A, B$ resp.

In the following let $T(X)^{+}$stand for $\{T(x)\}_{x \in X}^{+}$, for $X \subseteq A$. Before proving Theorem 1.2 we have:

LEMma 1.2. Let $\left\langle T, T^{*}\right\rangle$ be a Galois connection between the posets $A$ and $B$. Then $X_{1}^{\wedge+} \subseteq X_{2}^{\wedge+}$ in $\nu(A)$ implies $T\left(X_{2}\right)^{+} \subseteq T\left(X_{1}\right)^{+}$in $\nu(B)$.

Proof. We have

$$
X_{1}^{\wedge}=X_{1}^{\wedge+\wedge} \supseteq X_{2}^{\wedge+\wedge}=X_{2}^{\wedge}
$$

Let $y \in T\left(X_{2}\right)^{+}$. Since $y \leq T(x)$ for every $x \in X_{2}, x \leq T^{*}(y)$ for every $x \in$ $X_{2}$ follows. Thus, $T^{*}(y) \in X_{2}^{\wedge} \subseteq X_{1}^{\wedge}$. From $x \leq T^{*}(y)$ for every $x \in X_{1}$ we get $y \leq T(x)$, for $x \in X_{1}$ and $y \in T\left(X_{1}\right)^{+}$follows.

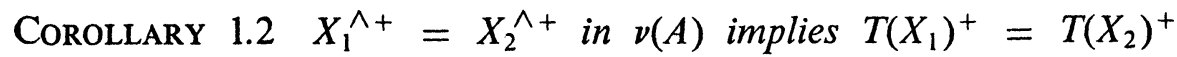
in $\nu(B)$.

Proof of Theorem 1.2. Identifying $A, B$ with their images in $\nu(A), \nu(B)$ we define $\tilde{T}: \nu(A) \rightarrow \nu(B)$ by

$$
\tilde{T}\left(X^{\wedge+}\right)=\bigwedge_{x \in X} \mathcal{F}(x), \quad X \subseteq A
$$

Here we put $T(0)=1, T(1)=0$ if 0,1 resp. are adjoined to $A$ and similarly for $T^{*}$. Since $\wedge_{x \in X} T(x)=T(X)^{+}$, it follows by Corollary 1.2 that $\tilde{T}$ is well-defined. By (1) $\tilde{T} \in \nu(A) \otimes \nu(B)$ since

$$
\begin{aligned}
\tilde{T}\left(\vee_{\alpha} X_{\alpha}{ }^{\wedge+}\right) & =\tilde{T}\left(\left(\cup_{\alpha} X_{\alpha}\right)^{\wedge+}\right)=\wedge_{x \in \cup_{\alpha} X_{\alpha}} T(x) \\
& =\wedge_{\alpha}\left(\wedge_{x \in X \alpha} T(x)\right)=\wedge_{\alpha} \tilde{T}\left(X_{\alpha}{ }^{\wedge+}\right) .
\end{aligned}
$$

Since $X^{\wedge+}=\vee_{x \in X} x$ for $X \subseteq A$, it follows by (1) that $\tilde{T}$ is the only possible extension of $T$. Obviously, $\tilde{T}^{*} \in \nu(B) \otimes \nu(A)$ is given by

$$
\tilde{T}^{*}\left(Y^{\wedge+}\right)=\bigwedge_{y \in Y} T^{*}(y), \quad Y \subseteq B .
$$

Corollary 2.2. $A \otimes B$ can be considered as a subset of $\nu(A) \otimes \nu(B)$. $\left\langle\tilde{T}, \tilde{T}^{*}\right\rangle$ is a Galois connection between $\nu(A)$ and $\nu(B)$ which is an extension of 
some Galois connection between $A$ and $B$ iff it satisfies (identifying, as before, $A$ and $B$ with their images in $\nu(A), \nu(B))$ :

$$
\begin{aligned}
& \text { for every } a \in A, \tilde{T}(a)=b_{0} \text { for some } b_{0} \in B \\
& \text { for every } b \in B, \tilde{T}^{*}(b)=a_{0} \text { for some } a_{0} \in A
\end{aligned}
$$

Since pseudo-complementation defines a Galois connection on a pseudo-complemented meet-semilattice $A$, Theorem 1.2 can be used to show that $\nu(A)$ is pseudo-complemented. In particular, a direct application of Theorem 1.2 yields the Glivenko-Stone Theorem, for the Boolean lattice $A$, with $\left(X^{\wedge+}\right)^{\prime}$, the complement of $X^{\wedge+}$ in $\nu(A)$ given by $\left\{x^{\prime}\right\}_{x \in X}^{+}=\left\{X^{\prime}\right\}^{+}$. We call $T: A \rightarrow B$ a polarized mapping if $T \in A \otimes B$. If $T: A \times B \rightarrow C$ is given put $T_{a}(b)=T_{b}(a)=T(a, b)$, for $a \in A, b \in B$. The mapping $T$, $T: A \times B \rightarrow C$ is bipolarized if the mappings $T_{a}: B \rightarrow C, T_{b}: A \rightarrow C$ are polarized for each $a \in A, b \in B$. Biresiduated mappings are defined analogously.

THEOREM 2.2 Each bipolarized mapping $T: A \times B \rightarrow C$ can be uniquely extended to a bipolarized mapping $\nu(T): \nu(A) \times \nu(B) \rightarrow \nu(C)$.

Proof. Identifying $A, B, C$, with their images in $\nu(A), \nu(B), \nu(C)$ we define $\nu(T): \nu(A) \times \nu(B) \rightarrow \nu(C)$ by:

$$
\nu(T)\left(X^{\wedge+}, Y^{\wedge+}\right)=\underset{\substack{x \in X \\ y \in Y}}{\wedge} T(x, y)
$$

where $T(0, a)=T(b, 0)=1, a \in A, b \in B$; if 1 has to be adjoined then $T(1, b)=T(a, 1)=0$ for $0 \neq a \in A, 0 \neq b \in B . T_{a}^{*}(c), T_{b}{ }^{*}(c)$, where either $a \in A, b \in B$ or $c \in C$ are universal are defined accordingly.

Using the equivalence of $T(x, y) \geq z$ with $T_{x}^{*}(z) \geq y$ and $T_{y}^{*}(z) \geq x$ it follows as in Lemma 1.2 and Corollary 1.2 that $\nu(T)$ is well-defined. As in Lemma 2.2 one can easily verify that both $\nu(T)_{X^{\wedge++}}: \nu(B) \rightarrow \nu(C), \nu(T)_{Y^{\wedge+}}$ : $\nu(A) \rightarrow \nu(C)$ are polarized for each $X^{\wedge+} \in \nu(A), Y^{\wedge+} \in \nu(B)$ with

$$
\nu(T)_{X \wedge+}^{*}\left(Z^{\wedge+}\right)=\bigwedge_{\substack{x \in X \\ z \in Z}} T_{x}^{*}(z), \quad Z \subseteq C
$$

$\nu(T)_{Y \wedge+}^{*}\left(Z^{\wedge+}\right)$ is given similarly. Consequently, $\nu(T)$ is bipolarized. $\nu(T)$ clearly extends $T$. The uniqueness of the extension can be shown using (1) as in Theorem 1.2. 
REMARK 1.2. Theorems 1.2 and 2.2 of course hold with "Galois connections" and "polarized mappings" replaced accordingly by either "residuated mappings" or "residual mappings." The extensions are then defined with appropriate dualizations.

A po-groupoid $(G, \cdot)$ is called residuated (see [1], [3], [9]) if the mapping $T: G \times G \rightarrow G$ given by $T\left(g_{1}, g_{2}\right)=g_{1} \cdot g_{2}, g_{1}, g_{2} \in G$, is biresiduated. This means that for every $a, b \in G$ there exist elements denoted by $a \cdot \cdot b$ (right residuals) and $a \cdot b$ (left residuals) such that $b \cdot x \leq a$ iff $x \leq a \cdot b$ while $x \cdot b$ $\leq a$ iff $x \leq a \cdot . b$. By Theorem 2.2 and Remark 1.2 we get:

COROLlary 3.2. The completion by cuts of a residuated (commutative) groupoid $G$ is a residuated (commutative) groupoid. In $\nu(G)$ multiplication is given by:

$$
X^{\wedge+} \cdot Y^{\wedge+}=\{x \cdot y \mid x \in X, y \in Y\}^{\wedge+}=(X \cdot Y)^{\wedge+}
$$

Right residuation in $\nu(G)$ is given by:

$$
X^{\wedge+} \cdot \cdot Y^{\wedge+}=\left\{m \cdot \cdot y \mid m \in X^{\wedge}, y \in Y\right\}^{+}=\left(X^{\wedge} \cdot Y\right)^{+}
$$

and similarly for left residuation. If 0 is adjoined to $G$ then $0 \cdot g=g \cdot 0=0$, $g \in G$; if 1 has to be adjoined we put $1 \cdot g=g \cdot 1=1,0 \neq g \in G$.

COROLlaRY 3*.2. The completion by cuts of a residuated semigroup is a residuated semigroup.

COROLlaRY 4.2. The conditional completion by nonvoid cuts of a directed residuated groupoid is a lattice-ordered residuated groupoid.

A lattice $A$ is called Brouwerian or relatively pseudo-complemented (see [1], p. 128) when $T: A \times A \rightarrow A$, given by $T(x, y)=x \wedge y, x, y \in A$ is a biresiduated mapping. We have:

Corollary 5.2. The completion by cuts of a Brouwerian lattice $A$ is a Brouwerian lattice.

This is an easy consequence of Corollary 3.2 where "." is replaced by " $\wedge$ " and both ".." and ".." by ":". It can be shown that $\wedge$ defined here coincides with the meet operation in $\nu(A)$, i.e., $X^{\wedge+} \cap Y^{\wedge+}=\{x \wedge y \mid x \in X$, $y \in Y\}^{\wedge+}=(X \wedge Y)^{\wedge+}$. Note that $1(=x: x) \in A$. 
A relation algebra $([1]$ p. 344$)$ is defined to be a residuated monoid $(G, \cdot)$ with 0 and unity $e$, which is a Boolean algebra when considered as a poset, in which $e^{\prime} \cdot g=e^{\prime} \cdot g$ holds for each $g \in G$, and denoting $e^{\prime} \cdot \cdot g^{\prime}$ by $\breve{g}$, the converse of $g$, such that $\breve{g}=g$ and $\bar{g} . h=\breve{h} . \bar{g}$ for $g, h \in G$. By using Theorem 2.2, the Glivenko-Stone Theorem, together with the natural definitions of complementation, multiplication and residuation in $\nu(G)$ we get:

COROLLARY 6.2. The completion by cuts of a relation algebra is a relation algebra, with conversion given by

$$
\breve{X}^{\wedge+}=\{\check{x}\}_{x \in X}^{\wedge+}=\check{X}^{\wedge+}
$$

3. Here we show that Galois connections between complete lattices $A$ and $B$ stand in a one-to-one correspondence with certain semi-ideals of $A \times B$.

Definition 1.3. Let $A, B$ be complete lattices. $\theta \subseteq A \times B$ is called a $G$-ideal of $A \times B$ when

(i) $(x, y) \leq(a, b)$ and $(a, b) \in \theta$ implies $(x, y) \in \theta$;

(ii) if $\left\{\left(a_{\alpha}, b_{\alpha}\right)\right\} \subseteq \theta$ then $\left(\vee_{\alpha} a_{\alpha}, \wedge_{\alpha} b_{\alpha}\right) \in \theta$ and $\left(\wedge_{\alpha} a_{\alpha}, \vee_{\alpha} b_{\alpha}\right) \in \theta$;

(iii) $(0,1) \in \theta$ and $(1,0) \in \theta$.

$K(A, B)$ denotes the set of all $G$-ideals of $A \times B$, partially ordered by inclusion. Since $A \times B \in K(A, B)$ and since $\cap_{\beta} \theta_{\beta}$, where $\left\{\theta_{\beta}\right\} \subseteq K(A, B)$ is easily shown to be a $G$-ideal of $A \times B$ we get:

LEMMA 1.3. $K(A, B)$ is a complete lattice.

Observe that in $K(A, B), \wedge_{\beta} \theta_{\beta}$, where $\theta_{\beta} \in K(A, B)$ satisfies:

$$
\wedge_{\beta} \theta_{\beta}=\left\{(x, y) \mid x=\wedge_{\beta} x_{\beta}, y=\wedge_{\beta} y_{\beta}, \quad \text { where }\left(x_{\beta}, y_{\beta}\right) \in \theta_{\beta}\right\} .
$$

We are going to prove:

TheOREM 1.3 $A \otimes B \cong K(A, B)$ when $A, B$ are complete lattices.

The proof of this theorem is based on the following two lemmas.

LEMMA 2.3. if $\left\langle T, T^{*}\right\rangle$ is a Galois connection between the complete lattices $A$ and $B$, then 


$$
\theta=\{(a, b) \mid T(a) \geq b\} \subseteq A \times B
$$

is a G-ideal.

Proof. If $(x, y) \leq(a, b)$ where $T(a) \geq b$, then $T(x) \geq T(a) \geq b \geq y$ since $T$ is antitone. If $T\left(a_{\alpha}\right) \geq b_{\alpha}$ for some set $\left\{\left(a_{\alpha}, b_{\alpha}\right)\right\} \subseteq A \times B$, then

$$
T\left(\vee_{\alpha} a_{\alpha}\right)=\wedge_{\alpha} T\left(a_{\alpha}\right) \geq \wedge_{\alpha} b_{\alpha} \text { by }(1)
$$

and

$$
T\left(\wedge_{\alpha} a_{\alpha}\right) \geq \vee_{\alpha} T\left(a_{\alpha}\right) \geq \vee_{\alpha} b_{\alpha}
$$

since $T$ is antitone. $T(0)=1$ by $(1)$ and $T(1) \geq 0$ is clear.

Lemma 3.3. Let $\theta \subseteq A \times B$ be a $G$-ideal of $A \times B$, where $A, B$ are complete lattices. If the mappings $T: A \rightarrow B, G: B \rightarrow A$ are defined by

$$
T(a)=\vee\{b \mid(a, b) \in \theta\} ; \quad G(b)=\vee\{a \mid(a, b) \in \theta\},
$$

then $\langle T, G\rangle$ is a Galois connection between $A$ and $B$.

Proof. T, $G$ are well-defined and antitone by (i), (iii) in Definition 1.3; (ii) implies that if $\left\{\left(a, b_{\alpha}\right)\right\} \subseteq \theta$ then $\left(a, \vee_{\alpha} b_{\alpha}\right) \in \theta$. Thus $(a, T(a)) \in \theta$ for each $a \in A$, and similarly, $(G(b), b) \in \theta$ for each $b \in B$. If $a \in A$ then $G T(a)=\vee\left\{a_{\alpha} \mid\left(a_{\alpha}, T(a)\right) \in \theta\right\} \geq a$. Also $T G(b) \geq b$ for each $b \in B$. Thus $\langle T, G\rangle$ is a Galois connection.

Observe that in Lemma $3.3(a, b) \in \theta$ is maximal in $\theta$ iff $a=G T(x), b$ $=T(x)$ for some $x \in A$.

Proof of Theorem 1.3. For every $T \in A \otimes B$ let $\sigma(T)=\theta$ be given by (5), and for every $\theta \in K(A, B)$ let $\eta(\theta)=T: A \rightarrow B$ be given by (6). By Lemmas 2.3, 3.3 $\sigma: A \otimes B \rightarrow K(A, B)$ while $\eta: K(A, B) \rightarrow A \otimes B$. For each $a \in A$ we have $\eta \sigma(T)(a)=\vee\{b \mid(a, b) \in \sigma(T)\}=\bigvee\{b \mid T(a) \geq b\}=T(a)$, hence $\eta \sigma(T)=T$ holds.

For $\theta \in K(A, B), \sigma \eta(\theta)=\{(a, b) \mid \eta(\theta)(a) \geq b\}=\left\{(a, b) \mid \vee_{\alpha}\left\{b_{\alpha} \mid\left(a, b_{\alpha}\right)\right.\right.$ $\in \theta\} \geq b\}$. If $(a, b) \in \theta$ then clearly $(a, b) \in \sigma \eta(\theta)$. Conversely, $(a, b) \in$ $\sigma \eta(\theta)$ implies $b \leq \vee_{\alpha} b_{\alpha}$ where $\left(a, b_{\alpha}\right) \in \theta$, and by (i), (ii) in Definition 1.3 $(a, b) \in \theta$. It follows that $\sigma \eta(\theta)=\theta . \sigma$ is thus one-to-one and onto with $\sigma^{-1}$ $=\eta$. Both $\sigma$ and $\eta$ are easily shown to be order-preserving and the proof is completed. 
4. Let $\Gamma \subseteq A \times B$ be given where $A, B$ are complete lattices. The minimal $G$-ideal $\theta$ of $A \times B$ which contains $\Gamma$ can be constructively described as follows: $\theta$ is the semi-ideal generated by the "closure" of $\{\Gamma$, $(0,1),(1,0)\}$ with respect to the property described in (ii) Definition 1.3. We call $\theta$ the $G$-ideal generated by $\Gamma$. Accordingly, the binary relation $\Gamma \subseteq$ $A \times B$ generates the Galois connection $\left\langle T, T^{*}\right\rangle$ between the complete lattices $A$ and $B$ if $\theta=\{(a, b) \mid T(a) \geq b\}$ is the $G$-ideal generated by $\Gamma$.

More generally, $\Gamma \subseteq A \times B$ generates the Galois connection $\left\langle T, T^{*}\right\rangle$ between the posets $A$ and $B$, if $\Gamma$ embedded in $\nu(A) \times \nu(B)$ generates $\langle\dot{\nu}(T)$, $\left.\nu\left(T^{*}\right)\right\rangle($ see $\S 2)$.

REMARK 1.4. It is important to observe that for every Galois connection $\left\langle T, T^{*}\right\rangle$ between the posets $A$ and $B$ there is at least one binary relation $\Gamma \subseteq A \times B$ generating it, namely, $\Gamma=\{(a, b) \mid T(a) \geq b\}$.

By Theorems 1.2, 1.3 we get:

Corollary $1.4 \Gamma \subseteq A \times B$ generates the Galois connection $\langle T, T$ * between the posets $A$ and $B$ iff $T$ is the minimal element of $A \otimes B$ which satisfies $T(a) \geq b$ for every $(a, b) \in \Gamma$.

For a given set $\Gamma \subseteq A \times B$ we determine the Galois connection $\langle T$, $\left.T^{*}\right\rangle$ between $\nu(A)$ and $\nu(B)$ generated by $\Gamma$, by constructing the minimal $G$-ideal of $\nu(A) \times \nu(B)$ which contains $\Gamma .\left\langle\tilde{T}, \tilde{T}^{*}\right\rangle$ may be restricted to a Galois connection between $A$ and $B$ in case it satisfies (3) (Corollary 2.2). By letting $\Gamma$ pass over all subsets of $A \times B$, all Galois connections between the posets $A$ and $B$ are determined.

The procedure presented here extends the one given by Birkhoff ([1], p. 122): If $\left\langle T, T^{*}\right\rangle$ is the Galois connection between $A=2^{M}$ and $B=2^{N}$ generated by $\rho \subseteq M \times N$ in Birkhoff's sense then $T$ is the minimal element of $A \otimes B$ satisfying $T(p) \geq q$ for each $(p, q) \in \rho$. Therefore (Corollary 1.4) $\left\langle T, T^{*}\right\rangle$ is also generated by $\rho$, embedded in $A \times B$, in our sense.

For completely distributive complete lattices we have:

LEMMA 1.4. Let $A, B$ be completely distributive, complete lattices. Then the G-ideal of $A \times B$ generated by $\Gamma \subseteq A \times B$ equals

$$
\theta=\left\{(x, y) \mid x \leq \bigwedge_{\alpha \in \Omega} \underset{\beta \in \mathrm{B}_{\alpha}}{\vee} a_{\alpha \beta}, y \leq \underset{\alpha \in \Omega}{\vee} \underset{\beta \in \mathrm{B}_{\alpha}}{\wedge} b_{\alpha \beta},\right\}
$$

where

$$
\left.\left(a_{\alpha \beta}, b_{\alpha \beta}\right) \in \Gamma \cup(0,1) \cup(1,0)\right\}
$$


Proof. Since $\theta$ is conained in each $G$-ideal which contains $\Gamma$, it suffices to show that $\theta$ is a $G$-ideal. (i), (iii) in Definition 1.3 are obvious, while (ii) can be easily proved by using the complete distributivity of both $A$ and $B$.

Corollary 2.4. If $\left\{\theta_{\alpha}\right\}, \alpha \in \Omega$, is a family of $G$-ideals of $A \times B$, where $A$ and $B$ are completely distributive complete lattices, then $\vee_{\alpha} \theta_{\alpha}$, the $G$-ideal generated by $\cup_{\alpha} \theta_{\alpha}$ satisfies

$$
\bigvee_{\alpha} \theta_{\alpha}=\left\{(x, y) \mid x \leq \bigwedge_{i \in I} \bigvee_{\alpha \in \Omega} a_{\alpha}^{i}, y \leqq \bigvee_{i \in I} \wedge_{\alpha \in \Omega} b_{\alpha}^{i},\right.
$$

$$
\text { where } \left.\left(a_{\alpha}^{i}, b_{\alpha}^{i}\right) \in \theta_{\alpha} \text { for each } i \in I\right\} \text {. }
$$

The results presented above can be applied to either residuated or residual mappings. In particular, every residuated (residual) mapping $T: A \rightarrow B, A, B$ complete lattices is uniquely determined by a set $\sigma(T)=\theta \subseteq A \times B$, namely, $\theta=\{(a, b) \mid T(a) \leq b\}(=\{(a, b) \mid T(a) \geq b\}$ resp.) such that:

(i) $(a, b) \in \theta$ and $x \leq a, y \geq b(x \geq a, y \leq b$ resp.) implies $(x, y) \in \theta$;

(ii) if $\left\{\left(a_{\alpha}, b_{\alpha}\right)\right\} \subseteq \theta$ then $\left(\vee_{\alpha} a_{\alpha}, \vee_{\alpha} b_{\alpha}\right) \in \theta$ and $\left(\wedge_{\alpha} a_{\alpha}, \wedge_{\alpha} b_{\alpha}\right) \in \theta$;

(iii) $(0,0) \in \theta,(1,1) \in \theta$.

Call $\theta \subseteq A \times B$ a $G$-relation if it satisfies unbracketed properties (i), (ii), (iii) listed above. The following observation was made by M. F. Janowitz. If $\theta_{1}=\sigma\left(T_{1}\right) \subseteq A \times B, \theta_{2}=\sigma\left(T_{2}\right) \subseteq B \times C$ are $G$-relations then $\sigma\left(T_{1}\right)$ - $\sigma\left(T_{2}\right)=\left\{(a, c) \mid(a, b) \in \sigma\left(T_{1}\right),(b, c) \in \sigma\left(T_{2}\right)\right.$ for some $\left.b \in B\right\}$ is a $G$-relation of $A \times C$, and $\sigma\left(T_{1}\right) \circ \sigma\left(T_{2}\right)=\sigma\left(T_{1} \cdot T_{2}\right)$. Consequently, $\sigma$ embeds the semigroup of residuated mappings on the complete lattice $A$ into the semigroup of binary relations on $A$.

5. We now study order-theoretic properties of $A \otimes B$. Firstly observe that $T_{1} \leq T_{2}$ in $A \otimes B$ iff $T_{1}^{*} \leq T_{2}^{*}$ in $B \otimes A$. This follows by the equivalence of $T(p) \geq q$ with $T^{*}(q) \geq p$. Since $\left(T^{*}\right)^{*}=T$ in $A \otimes B$ we have the well-known

TheOREM $1.5 A \otimes B \cong B \otimes A$, for any two posets $A, B$.

For $A, B$ bounded posets let the mapping $L_{b}^{a}: A \rightarrow B, a \in A, b \in B$ be defined by: 


$$
L_{b}^{a}(x)= \begin{cases}1 & x=0 \\ b & 0<x \leq a \\ 0 & x \ddagger a\end{cases}
$$

One can easily prove:

LEMmA 1.5. Let $A, B$ be bounded posets. Then: (i) $L_{b}^{a} \in A \otimes B$ with $\left(L_{b}^{a}\right)^{*}=L_{a}^{b}$ (ii) $T \in A \otimes B$ satisfies $T(a) \geq b$ iff $T \geq L_{b}^{a}$.

By Remark 1.4, Corollary 1.4, and Lemma 1.5 we get?

Theorem 2.5. Let $A, B$ be bounded posets. Every $T \in A \otimes B$ can be represented as a 1.u.b. of some set $\left\{L_{b_{\alpha}}^{a_{\alpha}}\right\} \subseteq A \otimes B, \alpha \in I$.

For complete lattices this result is independently proved in [11]. If $A, B$ are bounded posets, $A \otimes B$ is also bounded with $0=L_{o}^{a}=L_{b}^{o}, a \in A, b \in$ $B$ and $1=L_{1}^{1}$. The proof of the following lemma is straight-forward.

LeMma 2.5. Let $A, B$ be bounded posets. Then:

(i ) $L_{b}^{a} \leq L_{b}^{\hat{a}}$ iff $a \leq \hat{a} \in A, b \leq \hat{b} \in B$, when $a, b$ are non-zero;

(ii) if $\underset{\alpha}{\vee} a_{\alpha}=a, \underset{\beta}{\bigvee} b_{\beta}=b$ then $\underset{\alpha, \beta}{\vee} L_{b_{\beta}}^{a_{\alpha}}=L_{b}^{a}$;

(iii) if $\wedge_{\alpha} a_{\alpha}=a, \wedge_{\alpha} b_{\alpha}=b$ then $\wedge_{\alpha} L_{b_{\alpha}}^{a_{\alpha}}=L_{b}^{a}$.

Now put $L_{b}=L_{b}{ }^{1} \in A \otimes B$, where $b \in B$. By Lemma 1.5 we can put $L_{a}^{*}=L_{1}{ }^{a} \in A \otimes B$, where $a \in A$. Note that by Lemma 2.5:

then

$$
\text { if } \wedge_{\alpha} b_{\alpha}=b\left(\vee_{\alpha} b_{\alpha}=b\right) \text { in } B
$$

$$
\wedge_{\alpha} L_{b_{\alpha}}=L_{b}\left(\vee_{\alpha} L_{b_{\alpha}}=L_{b}\right) \text { in } A \otimes B
$$

A similar result holds for the mappings $L_{a}^{*}, a \in A$. Also

$$
L_{b}^{a}=L_{1 \wedge b}^{a \wedge 1}=L_{1}^{a} \wedge L_{b}^{1}=L_{a}^{*} \wedge L_{b}
$$

In connection with (9) note:

LeMmA 3.5 Let $A, B$ be bounded posets. The mapping $E_{b}^{a}: A \rightarrow B$, $a \in A, b \in B$, defined by:

$$
E_{b}^{a}(x)= \begin{cases}1 & x \leq a \\ b & x \notin a\end{cases}
$$


belongs to $A \otimes B$ with $\left(E_{b}^{a}\right)^{*}=E_{a}^{b}$ and

$$
E_{b}^{a}=L_{a}^{*} \vee L_{b}
$$

The proof is omitted. Using Lemma 2.5, (8), (9), (10) we conclude:

THEOREM 3.5. The mappings $a \rightarrow L_{a}^{*}, b \rightarrow L_{b}$ embed the bounded posets $A, B$ resp. in $A \otimes B$. Under these embeddings;

(i) 1.u.b.s. and g.1.b.s. are preserved;

(ii) $O_{A}$ and $O_{B}$ go into $O_{A \otimes B}, 1_{A}$ and $1_{B}$ go into $1_{A \otimes B}$;

(iii) if $a \in A, b \in B$ then their images have both $a$ l.u.b. and $a$ g.1.b.;

(iv) the images of different pairs $\left(a_{i}, b_{i}\right)$ possess different 1.u.b.s. and different g.1.b.s. provided that $a_{i} \in A, b_{i} \in B$ are not universal.

Corollary 1.5. $A \otimes 2 \cong A$, when $A$ is a bounded poset.

LEMMA 4.5. If $T=\wedge_{\alpha} L_{b_{\kappa}} \in A \otimes B$, with $A, B$ bounded posets, then $T$ $=L_{\wedge_{\alpha} b_{\alpha}}$

Proof. For each $0<x_{o} \in A, T\left(x_{o}\right) \leq L_{b_{\alpha}}\left(x_{o}\right)=b_{\alpha}$. Hence (Lemma 2.5 (ii)) $L_{T\left(x_{0}\right)}^{(x)} \leq L_{b_{\alpha}}$, and therefore $L_{T\left(x_{0}\right)} \leq T$. For each $0<x \neq x_{o}$ in $A$, $T\left(x_{o}\right)=L_{T\left(x_{o}\right)} \leq T(x)$. Similarily $T(x) \leq T\left(x_{o}\right)=b$ holds and $T=L_{b}$ follows. $b=\vee_{\alpha} b_{\alpha}$ is easily verified.

Note that by Lemma 4.5 and Theorem $1.5 T=\wedge_{\alpha} L_{a_{\alpha}}^{*}$ in $A \otimes B$ implies $T=L_{\wedge_{\alpha}}^{*} a_{\alpha}$.

6. It seems interesting to ask which properties of $A, B$ are inherited by $A \otimes B$. In [7] an example is given of two bounded lattices $A, B$ for which $A \otimes B$ is not a lattice. However, if $A, B$ are complete lattices so is $A \otimes B$ (Lemma 1.3). Actually, by using Lemma 4.5 and the remark after it we have:

TheOREM 1.6 Let $A, B$ be bounded posets. $A \otimes B$ is a complete lattice iff $A$ and $B$ are complete lattices.

We can also prove:

TheOREM 2.6. Let $A, B$ be bounded posets. $A \otimes B$ is a completely distributive complete lattice iff $A$ and $B$ are completely distributive complete lattices. 
Proof. The "only if" part follows by Theorem 1.6, (8) and the remark following (8). We now prove the "if" part. By Theorem 1.3 it suffices to show that $K(A, B)$, the complete lattice of $G$-ideals of $A \times B$, is completely distributive. Let $\left\{\theta_{\alpha \beta}\right\} \subseteq K(A, B)$ be given. Since

$$
\bigwedge_{\alpha \in \Omega} \underset{\beta \in B_{\alpha}}{\vee} \theta_{\alpha \beta} \supseteq \underset{\varphi \in \Pi \beta_{\alpha}}{\bigvee} \bigwedge_{\alpha \in \Omega} \theta_{\alpha \varphi(\alpha)}
$$

we have to prove the reverse inclusion. Assume $(x, y) \in \wedge_{\alpha \in \Omega} \vee_{\beta \in B_{\alpha}} \theta_{\alpha \beta}$. By (4) and (7) we have

$$
(x, y) \leq\left(\wedge_{\alpha \in \Omega} \wedge_{i \in I_{\alpha}} \vee_{\beta \in B_{\alpha}} x_{\alpha \beta}^{i}, \wedge_{\alpha \in \Omega} \vee_{i \in I_{\alpha}} \wedge_{\beta \in B_{\alpha}} y_{\alpha \beta}^{i}\right)
$$

where $\left(x_{\alpha \beta}^{i}, y_{\alpha \beta}^{i}\right) \in \theta_{\alpha \beta}$.

Using the complete distributivity of $A, B$ and the identity

$$
\wedge_{\alpha \in \Omega} \wedge_{\beta \in B_{\alpha}} a_{\alpha \beta}=\wedge_{\phi \in \Pi B_{\alpha}} \wedge_{\alpha \in \Omega} a_{\alpha \phi(\alpha)}
$$

we get:

$$
\begin{aligned}
& (x, y) \leq\left(\wedge_{\psi} \wedge_{\alpha \in \Omega} \vee_{\beta \in B_{\alpha}} x_{\alpha \beta}^{\psi(\alpha)}, \vee_{\psi} \wedge_{\alpha \in \Omega} \wedge_{\beta \in B_{\alpha}} y_{\alpha \beta}^{\psi(\alpha)}\right)= \\
& =\left(\wedge_{\psi} \vee_{\phi \in \Pi B_{\alpha}} \wedge_{\alpha \in \Omega} x_{\alpha \phi(\alpha)}^{\psi(\alpha)}, \vee_{\psi} \wedge_{\phi \in \Pi B_{\alpha}} \wedge_{\alpha \in \Omega} y_{\alpha \phi(\alpha)}^{\psi(\alpha)}\right)=(a, b)
\end{aligned}
$$

By (4); each

$$
\left(\wedge_{\alpha \in \Omega} x_{\alpha \phi(\alpha)}^{\psi(\alpha)}, \wedge_{\alpha \in \Omega} y_{\alpha \phi(\alpha)}^{\psi(\alpha)}\right)
$$

belongs to

$$
\bigwedge_{\alpha \in \Omega} \theta_{\alpha \phi(\alpha)}
$$

By (7) it now follows that

$$
(x, y) \leq(a, b) \in \vee_{\alpha \in \Pi B_{\alpha}} \wedge_{\alpha \in \Omega} \theta_{\alpha \phi(\alpha)},
$$

which completes the proof.

Corollary 1.6. If $A$ and $B$ are finite distributive lattices then $A \otimes B$ is distributive. 
Now let $T \in A \otimes B$ be given where $A$ and $B$ are completely distributive, complete lattices. By Theorem 2.5, (8) (9) (10) and Theorem 2.6 we get:

$$
\begin{gathered}
T=\vee_{\alpha} L_{b_{\alpha}}^{a_{\alpha}}=\vee_{\alpha}\left(L_{a_{\alpha}}^{*} \wedge L_{b_{\alpha}}\right) \\
=\wedge_{J \in 2 I}\left(\left(\vee_{\alpha \in J} L_{a_{\alpha}}^{*}\right) \vee\left(\vee_{\alpha \in I-J} L_{b_{\alpha}}\right)\right) \\
=\wedge_{J \in 2 I}\left(L_{\vee_{\alpha} \in J a_{\alpha}}^{*} \vee L_{\vee_{\alpha} \in I-J} b_{\alpha}\right) \\
=\bigwedge_{J \in 2^{I}}\left(L_{a_{J}}^{*} \vee L_{b_{J}}\right)=\bigwedge_{J \in 2 I} E_{b_{J}}^{a_{J}},
\end{gathered}
$$

(here $2^{I}$ is the power-set of $I, a_{J}=0$ if $J$ is empty and $b_{J}=0$ if $J=I$ ). Therefore $\sigma(T)$, the $G$-ideal of $A \times B$ associated with $\left\langle T, T^{*}\right\rangle$ equals $\{(x$, $y) \mid$ either $x \leq a_{J}$ or $y \leq b_{J}$ for each $\left.J\right\}$. This means that $\left\langle T, T^{*}\right\rangle$ is a tight Galois connection ([13]). (11) relates our characterization of Galois connections to the one given by Raney for completely distributive complete lattices.

We now prove:

THEOREM 3.6. $A \otimes 2^{B} \cong A^{B}$ when $A$ is a complete lattice and $B$ a poset. By [1] p. 56, the complete lattice $2^{B}$ of all isotone mappings $T: B \rightarrow 2$ is isomorphic to the set of all dual semi-ideals of $B$, partially ordered by inclusion. Under this isomorphism $B_{f}=\{x \mid x \in B, f(x)=1\}$ corresponds to $f \in 2^{B} \cdot f_{b} \in 2^{B}$ is the characteristic function of $\{x \mid x \geq b\} \subseteq B$, i.e., $f_{b}(x)=1$ iff $x \geq b \in B$.

Proof of Theorem 3.6. It suffices to prove that $2^{B} \otimes A \cong A^{B}$ (Theorem 1.5). For each $t \in 2^{B} \otimes A$ let $\sigma(t): B \rightarrow A$ be defined by $\sigma(t)(b)=t\left(f_{b}\right)$. Since $b \leq b$ in $B$ iff $f_{b} \geq f_{b}$ in $2^{B}$ and since $t: 2^{B} \rightarrow A$ is antitone, it follows that $\sigma(t)$ is isotone, i.e., $\sigma: 2^{B} \otimes A \rightarrow A^{B}$. For every $T \in A^{B}$ let $\eta(T): 2^{B} \rightarrow$ $A$ be defined by

$$
\eta(T)(f)=\bigwedge_{b \in B_{f}} T(b), f \neq 0 \text { in } 2^{B} ; \quad \eta(T)(0)=1 .
$$

If $\left\{f_{\alpha}\right\} \subseteq 2^{B}$ then

$$
\begin{aligned}
& \eta(T)\left(\underset{\alpha}{\bigvee} f_{\alpha}\right)=\underset{b \in \cup_{\alpha} B_{f_{\alpha}}}{\bigwedge} T(b) \\
= & \bigwedge_{\alpha} \bigwedge_{b \in B_{f_{\alpha}}} T(b)=\bigwedge_{\alpha} \eta(T)\left(f_{\alpha}\right) .
\end{aligned}
$$


By (1), $\eta(T) \in 2^{B} \otimes A$ follows, hence $\eta: A^{B} \rightarrow 2^{B} \otimes A$. For every $T \in A^{B}$ and $b \in B, \sigma \eta(T)(b)=\eta(T)\left(f_{b}\right)=\wedge_{b^{\prime} \geq b} T\left(b^{\prime}\right)=T(b)$; thus $\sigma \eta(T)=T$. Conversely, for each $t \in 2^{B} \otimes A$, and $f \neq 0$ we get, using (1):

$$
\sigma \eta(t)(f)=\wedge_{b \in B_{f}} \sigma(t)(b)=\wedge_{b \in B_{f}} t\left(f_{b}\right)=T\left(\vee_{b \in B_{f}} f_{b}\right)=t(f)
$$

Since $\eta \sigma(t)(0)=1=t(0)$, we proved that $\eta \sigma(t)=t$. Consequently, $\sigma$ is one-to-one and onto with $\sigma^{-1}=\eta$. $\sigma$ and $\eta$ are easily shown to be isotone, hence $A^{B}$ and $2^{B} \otimes A$ are isomorphic.

Theorem 3.6 yields:

COROLlary 2.6. $2^{M} \otimes 2^{N} \cong 2^{M \times N}$, when $M, N$ are posets.

If both $M, N$ are totally disordered posets, $2^{M}, 2^{N}$ are the lattices of all subsets of $M, N$ resp., and by Corollary $2.6,2^{M} \otimes 2^{N}$ is the lattice of all binary relations $\rho \subseteq M \times N$ (this is exactly Birkhoff's and Everett's characterization). By Tarsk:'s Theorem ([1] p. 119) this proves the "if" part of

TheOREM 4.6. Let $A, B$ be bounded posets. $A \otimes B$ is a completely distributive complete Boolean lattice iff $A$ and $B$ are completely distributive Boolean lattices.

Proof of the "only if" part. Assume $A \otimes B$ is a completely distributive complete Boolean lattice. By Theorems 1.6, 2.6, and by symmetry it suffices to prove that $A$ is complemented. For $a \in A$ let $T=\mathrm{V}_{\alpha} L_{b_{\alpha}}^{a}$ be the complement of $L_{a}^{*}$ in $A \otimes B$. (We may assume $b_{\alpha} \neq 0$ for each $\alpha$ ). By (9),

$$
0=L_{a}^{*} \wedge \vee_{\alpha} L_{b_{\alpha}}^{a_{\alpha}}=\vee_{\alpha} L_{b_{\alpha}}^{a \wedge a_{\alpha}}
$$

thus $a \wedge a_{\alpha}=0$ and $a \wedge \vee_{\alpha} a_{\alpha}=0$ follows. Since $L_{a}^{*}=L_{1}^{a}$, we get (see (11)):

$$
1=L_{1}^{a} \vee \vee_{\alpha} L_{b_{\alpha}}^{a_{\alpha}}=\wedge_{J \in 2 I} E_{b_{J}}^{a J}
$$

Taking $J=I, E_{0}^{a \vee \vee_{\alpha} a_{\alpha}}=1$ follows. Hence $a \vee \vee_{\alpha} a_{\alpha}=1$, and $\vee_{\alpha} a_{\alpha}$ is the complement of $a$.

7. We discuss here some formal properties of $\otimes$ as a commutative binary operation on posets. Using the $G$-ideal characterization of $A \otimes B$ we have: 
Theorem 1.7. $A \otimes(B \otimes C) \cong(A \otimes B) \otimes C$, when $A, B, C$ are complete lattices. ideas.

This theorem is independently proved in [11] using basically different

Proof. Let $A \otimes B \otimes C$ denote the set of all subsets $Y \subseteq A \times B \times C$ satisfying:

(i ) $(x, y, z) \leq(a, b, c)$, and $(a, b, c) \in Y$ implies $(x, y, z) \in Y$;

(ii) $\left\{\left(a_{\alpha}, b_{\alpha}, c_{\alpha}\right)\right\} \subseteq Y$ implies

$$
\begin{aligned}
& \left(\vee_{\alpha} a_{\alpha}, \wedge_{\alpha} b_{\alpha}, \wedge_{\alpha} c_{\alpha}\right) \in Y, \\
& \left(\wedge_{\alpha} a_{\alpha}, \vee_{\alpha} b_{\alpha}, \wedge_{\alpha} c_{\alpha}\right) \in Y, \\
& \left(\wedge_{\alpha} a_{\alpha}, \wedge_{\alpha} b_{\alpha}, \vee_{\alpha} c\right) \in Y ;
\end{aligned}
$$

(iii) $(0,1,1) \in Y,(1,0,1) \in Y,(1,1,0) \in Y$. Order $A \otimes B \otimes C$ by inclusion. For any $X \in A \otimes(B \otimes C)$ let $\sigma(X)=\{(a, b, c) \mid(b, c) \in$ $\theta$, and $(a, \theta) \in X$ for some $\theta \in B \otimes C\} . \sigma(X) \in A \otimes B \otimes C$ is easily verified. If $Y \in A \otimes B \otimes C$, let $Y(a)=\{(b, c) \mid(a, b, c) \in Y\} \in B \otimes C$ and put

$$
\eta(Y)=\{(a, \theta) \mid a \in A, \theta \in B \otimes C, \text { and } \theta \subseteq Y(a)\} .
$$

$\eta(Y) \in A \otimes(B \otimes C)$ is easily shown. $\sigma$ and $\eta$ are clearly isotone. By using the definitions we get $\eta \sigma(X)=X, \sigma \eta(Y)=Y$, for each $X \in A \otimes(B \otimes C)$, $Y \in A \otimes B \otimes C$. Hence

$$
A \otimes(B \otimes C) \cong A \otimes B \otimes C .
$$

$(\mathrm{A} \otimes B) \otimes C \cong A \otimes B \otimes C$ can be shown in a similar manner Using Theorem 1.7, 3.6 and 1.5 we get the interesting:

Corollary 1.7. $A \otimes B^{C} \cong A^{C} \otimes B \cong(A \otimes B)^{C}$ where $A, B$ are complete lattices and $C$ is a poset.

(this corollary together with Theorems $1.5,3.6,1.7$ suggests the formal notation $A \otimes B \equiv A^{\log _{2} B}$ ).

Let $\Pi_{\alpha} A_{\alpha}$ denote the Cartesian product of the posets $A_{\alpha}$, partially ordered pointwise. We then have:

THEOREM 2.7. $A \otimes \Pi_{\alpha} B_{\alpha} \cong \Pi_{\alpha}\left(A \otimes B_{\alpha}\right)$ when $A$ is a complete lattice and the $B_{\alpha}$ are bounded posets. If the number of posets $B_{\alpha}$ is finite, it suffices that $A$ be a bounded lattice. 
Proof. If $T \in A \otimes \Pi_{\alpha} B_{\alpha}$ then $T^{\alpha}: A \rightarrow B_{\alpha}$ defined by

$$
T^{\alpha}(a)=\{T(a)\}_{\alpha}, a \in A,
$$

belongs to $A \otimes B_{\alpha}$ with $\left(T^{\alpha}\right)^{*}\left(b_{\alpha}\right)=T^{*}\left(0,0, \ldots, b_{\alpha}, 0, \ldots\right), b_{\alpha} \in B_{\alpha}$. Let

$\sigma: A \otimes \Pi_{\alpha} B_{\alpha} \rightarrow \Pi_{\alpha}\left(A \otimes B_{\alpha}\right)$ be defined by $[\sigma(T)]_{\alpha}=T^{\alpha}$. Conversely, if $t \in \Pi_{\alpha}\left(A \otimes B_{\alpha}\right)$ then $\eta(t): A \rightarrow \Pi_{\alpha} B_{\alpha}$ defined by $[\eta(t)(a)]_{\alpha}=t_{\alpha}(a), a \in A$, belongs to $A \otimes \Pi_{\alpha} B_{\alpha}$ where

$$
\eta(t)^{*}\left(b_{1}, b_{2}, \ldots, b_{\alpha}, \ldots\right)=\wedge_{\alpha} t_{\alpha}^{*}\left(b_{\alpha}\right)
$$

for each $\left(b_{1}, b_{2}, \ldots, b_{\alpha}, \ldots\right) \in \Pi_{\alpha} B_{\alpha}$. One easily verifies that $\sigma$ induces the isomorphism between $A \otimes \Pi_{\alpha} B_{\alpha}$ and $\Pi_{\alpha}\left(A \otimes B_{\alpha}\right)$ where $\sigma^{-1}=\eta$.

A certain formal similarity can be noticed between properties of $A \otimes B$ as a product of two posets $A$ and $B$ and those of the tensor product of two linear spaces, with $L_{b}^{a}$ playing the part of the generators $a \otimes b$ (Theorems $2.5,1.2,1.7,2.7$, and Lemma 2.5 ). This similarity can be carried further by:

LEMMA 1.7. For every bipolarized mapping $T: A \times B \rightarrow C$ (see §2), where $A, B, C$ are complete lattices, there exists a unique polarized mapping $t: A \otimes B \rightarrow C$, such that $t\left(L_{b}^{a}\right)=T(a, b)$, for each $a \in A, b \in B$.

This lemma also appears independently in [11] with a different proof.

Proof. For each $G=\vee_{\alpha} L_{b_{\alpha}}^{a_{\alpha}} \in A \otimes B$ put $t(G)=\wedge_{\alpha} T\left(a_{\alpha}, b_{\alpha}\right)$. Since $T(0, b)=T(a, 0)=1$ for each $b \in B, a \in A$, and since

$$
T\left(\vee_{i} a_{i}, \wedge_{i} b_{i}\right)=\wedge_{i} T\left(a_{i}, \wedge_{i} b_{i}\right) \geq \wedge_{i} T\left(a_{i}, b_{i}\right)
$$

holds for every subset $\left\{\left(a_{i}, b_{i}\right)\right\} \subseteq A \times B$ it follows that

$$
\begin{aligned}
& \wedge_{\alpha} T\left(a_{\alpha}, b_{\alpha}\right)=\wedge\{T(a, b) \mid(a, b) \in \theta, \\
&\text { the } \left.G \text {-ideal of } A \times B \text { generated by }\left\{\left(a_{\alpha}, b_{\alpha}\right)\right\}\right\} .
\end{aligned}
$$

Thus $t: A \otimes B \rightarrow C$ is well defined, with $t\left(L_{b}^{a}\right)=T(a, b), a \in A, b \in B$. By (1) one easily verifies that $t \in(A \otimes B) \otimes C$. (1) also implies the uniqueness of $t$.

Lemma 1.7 together with Theorem 2.2 yield:

THEOREM 3.7. For every bipolarized mapping $T: A \times B \rightarrow C$, where $A$, 
$B, \quad C$ are posets there exists a unique polarized mapping $t: \nu(A) \otimes \nu(B) \rightarrow \nu(C)$ such that $t\left(L_{b}^{a}\right)=T(a, b)$ for each $a \in A \subseteq \nu(A), b \in B \subseteq \nu(B)$.

Since $T: A \rightarrow B$ is residuated if $T \in A \otimes B^{D}$ it follows that Theorem 3.7 holds also with "polarized" replaced by "residuated."

\section{REFERENCES}

1. G. Birkhoff, Lattice Theory, 3rd edition, AMS Colloquium Publications, Rhode Island, 1967.

2. J. C. Derdérian, Residuated mappings, Pacific J. Math. 20 (1967), 35-43.

3. P. Dubreil and R. Croisot, Propriétés générales de la résiduation, Collectana Mathematica 7 (1954), 193-203.

4. C. J. Everett, Closure operations and Galois theory in lattices, Trans. Amer. Math. Soc. 55 (1944), 514-525

5. M. F. Janowitz, A semigroup approach to lattices, Canad. J. Math. 18 (1966), 1212-1223. 6. __ On decreasing Baer semigroups, Bull. Soc. Roy. Sci. de Liége 38 (1969), $414-423$.

7. C. S. Johnson, Jr., A lattice whose residuated maps do not form a lattice, J. Nat. Sci. and Math. 9 (2) (1969), 283-284.

8. $\longrightarrow$ Semigroups coordinatizing posets and semilattices, J. London Math. Soc. (2) 4 (1971), 277-283.

9. L. O'Carrol, A basis for the theory of residuated groupoids, J. London Math. Soc. (2) 3 (1971), 7-20.

10. O. Ore, Galois connexions, Trans. Amer. Math. Soc. 55 (1944), 493-515.

11. D. G. Mowat, Ph.D. Thesis, University of Waterloo, 1968.

12. G. Pickert, Bemerkungen uber Galois-Verbindungen, Archiv. Math. 3 (1952), 385-389.

13. G.N. Raney, Tight Galois connections and complete distributivity, Trans. Amer. Math. Soc. 97 (1960), 418-426.

14. G. C. Rota, On the foundations of combinatorial theory. I. Theory of Mabius functions, Zeits. f. Wahrscheinlichkeitstheorie 2 (1964), 340-368.

15. A. Waterman, Rough notes taken by Henry Crapo at a lecture at McMaster University, 1967.

16. O. Wyler, TOP categories and categorical topology, General Topology and its Applications 1 (1971), 17-28.

Received March 12, 1973 and in revised form April 21,1974. This research was supported by a fellowship from Technion, Israel Institute of Technology, and is partially based on the author's D.Sc. thesis. The author wishes to thank Professor A. Ginzburg for his guidance and help.

'The author has learned that a similar observation was made independently by Alan Waterman [15] and also appears in [11], although, as it seems, these have never been published. 



\section{PACIFIC JOURNAL OF MATHEMATICS}

\section{EDITORS}

RICHARD ARENS (Managing Editor)

University of California

Los Angeles, California, 90024

\section{R. A. BEAUMONT}

University of Washington

Seattle, Washington 98105

\section{J. DugunDJI}

Department of Mathematics University of Southern California Los Angeles, California 90007

D. Gillbarg AND J. MilgRAM

Stanford University Stanford, California 94305

\section{ASSOCIATE EDITORS}

E. F. BECKENBACH

B. H. NEUMANN

SUPPORTING

UNIVERSITY OF BRITISH COLUMBIA

UNIVERSITY OF CALIFORNIA

MONTANA STATE UNIVERSITY

UNIVERSITY OF NEVADA

NEW MEXICO STATE UNIVERSITY

OREGON STATE UNIVERSITY

UNIVERSITY OF OREGON OSAKA UNIVERSITY
F. WOLF

K. YosHIDA

INSTITUTIONS

UNIVERSITY OF SOUTHERN CALIFORNIA

STANFORD UNIVERSITY

UNIVERSITY OF TOKYO

UNIVERSITY OF UTAH

WASHINGTON STATE UNIVERSITY

UNIVERSITY OF WASHINGTON

AMERICAN MATHEMATICAL SOCIETY

NAVAL WEAPONS CENTER

The Supporting Institutions listed above contribute to the cost of publication of this Journal, but they are not owners or publishers and have no responsibility for its content or policies.

Mathematical papers intended for publication in the Pacific Journal of Mathematics should be in typed form or offset-reproduced, (not dittoed), double spaced with large margins. Underline Greek letters in red, German in green, and script in blue. The first paragraph or two must be capable of being used separately as a synopsis of the entire paper. Items of the bibliography should not be cited there unless absolutely necessary, in which case they must be identified by author and Journal, rather than by item number. Manuscripts, in duplicate if possible, may be sent to any one of the five editors. Please classify according to the scheme of Math. Rev. Index to Vol. ${ }^{39}$. All other communications to the editors should be addressed to the managing editor, or Elaine Barth, University of California, Los Angeles, California, 90024.

100 reprints are provided free for each article, only if page charges have been substantially paid. Additional copies may be obtained at cost in multiples of 50 .

The Pacific Journal of Mathematics is issued monthly as of January 1966. Regular subscription rate: $\$ 72.00$ a year (6 Vols., 12 issues). Special rate: $\$ 36.00$ a year to individual members of supporting institutions.

Subscriptions, orders for back numbers, and changes of address should be sent to Pacific Journal of Mathematics, 103 Highland Boulevard, Berkeley, California 90708.

\section{PUBLISHED BY PACIFIC JOURNAL OF MATHEMATICS, A NON-PROFIT CORPORATION}

Copyright (C) 1974 by Pacific Journal of Mathematics

Manufactured and first issued in the U.S.A. 


\section{Pacific Journal of Mathematics}

\section{Vol. 54, No. $2 \quad$ June, 1974}

John Edward Coury, Walsh series with coefficients tending monotonically to

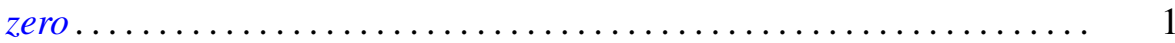

Patrick Michael Fitzpatrick and Walter Volodymyr Petryshyn, Fixed point theorems for multivalued noncompact acyclic mappings ............

Irving Leonard Glicksberg, More on Phragmén-Lindelöf for function

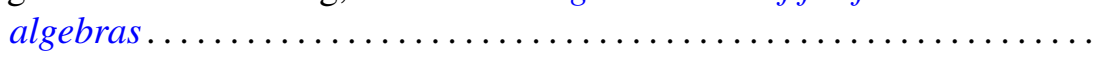

Adilson Goncalves, Structural constants. II .................. 39

Richard P. Gosselin, Closure theorems for affine transformation groups .... 53

Ralph Peter Grimaldi, Baer and UT-modules over domains ........... 59

Edward Grossman, On the prime ideal divisors of $\left(a^{n}-b^{n}\right) \ldots \ldots \ldots \ldots . \ldots 73$

A. Hedayat and Ester Seiden, On the theory and application of sum composition of Latin squares and orthogonal Latin squares.......... .

Gerald L. Itzkowitz, Continuous measures, Baire category, and uniform continuity in topological groups ......................... 115

Francis Masat, Right simple congruences on a semigroup ............ 127

Robert Harvey Oehmke, Right congruences and semisimplicity for Rees matrix semigroups..................................

Qazi Ibadur Rahman and Jan Stankiewicz, Differential inequalities and local valency . . . . . . . . . . . . . . . . . . . . . . . . . . . . . . . . . . . . . 165

William John Reed, Random points in a simplex ................ 183

Mohan S. Shrikhande, Strongly regular graphs and group divisible

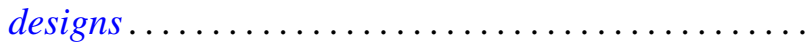

Zahava Shmuely, The structure of Galois connections ... . .

Robert C. Shock, Dual generalizations of the Artinian and Noetherian

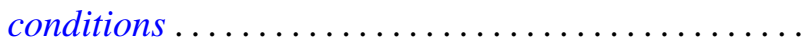

Arne Stray, Approximation and interpolation for some spaces of analytic functions in the unit disc ........................

Eldon Jon Vought, Monotone decompositions into trees of Hausdorff continua irreducible about a finite subset ............

James Wirth, The mapping cylinder axiom for WCHP fibrations ... 263

Gordon S. Woodward, Invariant means and ergodic sets in Fourier analysis... 